\title{
Endoglin (CD105) and vascular endothelial growth factor as prognostic markers in colorectal cancer
}

\author{
Reda S Saad ${ }^{1}$, Yulin L Liu², Girija Nathan², James Celebrezze ${ }^{3}$, David Medich ${ }^{3}$ and \\ Jan F Silverman ${ }^{2}$ \\ ${ }^{1}$ Department of Pathology, Louisiana State University Health Science Center, New Orleans, LA, USA; \\ ${ }^{2}$ Department of Pathology and ${ }^{3}$ Department of Surgery, Allegheny General Hospital, Pittsburgh, PA, USA
}

\begin{abstract}
Endoglin (CD105) has been shown to be a more useful marker to identify proliferating endothelium involved in tumor angiogenesis than panendothelial markers such as CD31. We investigated endoglin and vascular endothelial growth factor expression as possible prognostic markers in colorectal cancer. Surgical specimens from 150 patients with resected colorectal carcinomas were immunostained for endoglin, CD31 and vascular endothelial growth factor. Colorectal carcinoma cases consisted of 50 cases without lymph node metastases, 50 cases with only lymph node metastases and 50 cases with liver metastases (38 cases also had positive lymph nodes). Positively stained microvessels were counted in densely vascular foci (hot spots) at $\times 400$ fields in each specimen. For vascular endothelial growth factor, intensity of staining was scored on a three-tiered scale. Results were correlated with other prognostic parameters. Endoglin demonstrated significantly more proliferating neoplastic microvessels than CD31 (31 \pm 10 vs $19 \pm 8 / 0.15 \mathrm{~mm}^{2}$ field, $\left.P<0.001\right)$. Low vascular endothelial growth factor expression within tumor cells was seen in 49 (33\%) and high expression in 101 cases $(67 \%)$. There was a positive correlation of endoglin, CD31 counts and vascular endothelial growth factor overexpression with the presence of angiolymphatic invasion and lymph node metastases $(P<0.05)$. Only endoglin counts correlated significantly with liver metastases and positive vascular pedicle lymph nodes $(P<0.05)$, while vascular endothelial growth factor showed significant correlation with the depth of invasion $(P<0.01)$. Endoglin, by staining higher numbers of the proliferating vessels in colon carcinoma, is a more specific and sensitive marker for tumor angiogenesis than the commonly used panendothelial markers. Endoglin staining also showed prognostic significance with positive correlation with angiolymphatic invasion and metastases to lymph nodes and liver.
\end{abstract}

Modern Pathology (2004) 17, 197-203, advance online publication, 5 December 2003; doi:10.1038/modpathol.3800034

Keywords: endoglin; cancer colon; CD31; angiogenesis

In colorectal cancer patients, conventional prognostic parameters such as tumor grade, depth of penetration, angiolymphatic invasion and lymph node involvement are important prognostic factors. ${ }^{1-4}$ However, these histopathologic characteristics do not always predict the outcome for any individual patients, prompting the need for additional prognostic markers that can predict the outcome for these patients more accurately.

Tumor angiogenesis, the formation of peritumor and intratumor new blood vessels, is necessary to the growth and metastasis of solid tumors, and is regarded as one of the most important events occu-

Correspondence: R Saad, Department of Pathology, Louisiana State University Health Science Center, School of Medicine, 1901 Perdido Street, P5-1, New Orleans, LA 70112, USA.

E-mail: redasaad2@hotmail.com

Received 8 May 2003; revised 15 September 2003; accepted 23

September 2003; published online 5 December 2003 rring in the neoplastic process. ${ }^{3,5}$ Neovascularization allows tumor growth by supplying nutrient and oxygen, disposing metabolites and releasing growth factors that promote tumor cell proliferation. ${ }^{6}$ Neoplasms promote angiogenesis by secreting growth factors such as vascular endothelial growth factor (VEGF), hepatocytes growth factor and plateletderived growth factor that stimulate endothelial migration and proliferation. ${ }^{7,8}$ Angiogenesis also contributes to the metastatic process by providing large numbers of leaking blood vessels for vascular invasion. ${ }^{7}$

Recently, there is growing evidence supporting microvessel density as an important predictor of tumor behavior in a number of human malignancies. $^{9-16}$ The role of angiogenesis in colorectal tumors has also been investigated, but the literature shows contradictory results. While Bossi et $a 1^{17}$ and Pietra et $a 1^{18}$ reported that angiogenesis did not provide any significant prognostic information for colorectal cancer patients, Saclarides et $a l^{19}$ and 
Frank et $a l,{ }^{20}$ using factor VIII, reported a significant correlation of increased vascularization of colorectal carcinomas with recurrence, metastasis and survival.

Endoglin (CD105) is a receptor for the TGF- $\beta 1$ molecule that is upregulated in tumor neoangiogenesis. ${ }^{21}$ Endoglin, as a marker for neoangiogenesis, has certain advantages over the other commonly used panendothelial markers (CD31, CD34, Factor VIII). The endoglin antibody binds preferentially to the activated endothelial cells that participate in tumor angiogenesis, ${ }^{22}$ but expression is weak/or negative in vascular endothelium of normal tissues. ${ }^{23,24}$ Recently, several studies have examined endoglin expression in cancers of the endometrium, cervix and breast, ${ }^{21,22,24}$ and found endoglin as a more specific and sensitive microvessel marker than other commonly used panendothelial antibodies.

The purpose of this retrospective study is to compare CD31, a panendothelial marker commonly used to determine microvessel density, with endoglin (CD105). We also evaluated the VEGF expression in colorectal cancer. We then correlated the results of microvessel counts with CD31 and endoglin and VEGF expression with other standard prognostic parameters such as size, grade, depth of invasion, angiolymphatic invasion, and lymph nodes and liver metastases.

\section{Material and methods}

Clinical data and pathologic tissue samples were retrieved from the computer files at the Allegheny General Hospital, Department of Pathology, from January 1998 to December 2000. A total of 150 cases of unselected patients with colorectal cancer that were surgically treated with total colectomy and vascular pedicle lymph node resection were included in this study. Computerized tomography (CT) scanning was performed in all cases as part of clinical staging. Cases with liver lesions were subjected to surgical biopsy to confirm the diagnosis of metastases.

Tissues from the specimens were fixed in 10\% buffered-formalin, processed and stained with hematoxylin and eosin. Three investigators (RSS, YL and GN) reviewed all cases to confirm the diagnosis. One paraffin block with the maximum bulk of tumor and maximum depth of invasion was chosen from each case for immunohistochemical studies. Majority of slides $(127 / 150,84 \%)$ showed the nonneoplastic colonic tissue-carcinoma junction.

\section{Immunohistochemistry}

Sections, $4 \mu \mathrm{m}$ thick from the paraffin-embedded tissue blocks were stained for endoglin, CD31 and VEGF. The tissue sections were mounted on coated slides and dried $1 \mathrm{~h}$ at $60^{\circ} \mathrm{C}$. The sections were deparaffinized in xylene and rehydrated in a descending ethanol series. The primary antibodies used were: mouse antihuman monoclonal antibody
(Clone SN6H, Dako, Carpinteria, CA, USA) at a 1:900 dilution for endoglin, a mouse monoclonal antibody (Clone JC/70A, Cell Marque, Hot Springs, AR, USA) for CD31 and a rabbit polyclonal antibody (Santa Cruz Biotechnology, Santa Cruz, CA, USA) at a 1:500 dilution for VEGF. The Ventana Immunostainer and basic DAB detection system (Ventana Medical Systems, Tucson, AZ, USA) were used for CD31 and VEGF staining. Endoglin staining was carried out by manual incubation of primary antibody overnight at $4^{\circ} \mathrm{C}$, then completed with the Ventana's High Intensity DAB Detection Kit. Appropriate positive and negative controls were run for each batch of slides.

\section{Quantitation and Statistics}

After scanning the immunostained section at low magnification $(\times 40)$, three areas of carcinoma with the greatest number of distinctly highlighted microvessels (hotspots) were selected by two observers (RSS and YL) at the same time. The two observers then independently evaluated the slides for microvessel counting using $\times 400$ magnification $\left(0.15 \mathrm{~mm}^{2}\right.$ field) without knowledge of patient status and stains used. Any single cell or spot that stained by the immunohistochemical marker was counted as a vessel. As in a previous report, a visible vascular lumen was not required to count as a microvessel. ${ }^{25}$ The greatest number of vessels counted was recorded and used in the statistical analysis. $\kappa$-value between the two observers was moderate (0.75). Discordant cases were recounted and consensus resolved any discrepancy more than $10 \%$ of the microvessel count. For VEGF, staining intensity was recorded as: 0 negative (no or $<20 \%$ of positive cells showing weak positivity), $1+$ weak ( $>20 \%$ of cells showing weak positivity), $2+$ strong ( $>20 \%$ showing strong positivity), with a moderate $\kappa$-value (0.76). Clinical data including tumor grade, depth of invasion, angiolymphatic invasion, and lymph node and liver metastases were correlated with microvessel count. Correlation with clinical data was evaluated by Spearman ' $p$ ' correlation and multivariate analysis. Mean differences in microvessel counts were compared with the use of paired ' $t$ ' tests. All statistical analyses were performed with SPSS (Statistical Package for Social Sciences, SPSS Inc., Chicago, IL, USA).

\section{Results}

Table 1 shows the results of our study.

\section{Patients with Negative Lymph Nodes}

This group included 35 female and 15 male patients with a mean age of $72 \pm 9$ years and a range of $40-88$ years. The anatomic location of the resected tumors 
included 28 cases in right colon, six in transverse colon and 16 in sigmoid colon. The mean number of lymph nodes retrieved per case was $20 \pm 8$ with a mode of 11 lymph nodes. Histologically, 41 cases were moderately differentiated, five poorly differentiated and four well differentiated. Angiolymphatic invasion was not identified in any of these patients. The mean microvessel density value for endoglin was $26 \pm 10 / 0.15 \mathrm{~mm}^{2}$ field, $15 \pm 8$ / $0.15 \mathrm{~mm}^{2}$ field for CD31 and $1.6 \pm 1.1$ for VEGF. Paired ' $t$ ' test showed a significant difference between endoglin and CD31 microvessel counts $(P<0.001)$. However, there was a significant correlation between endoglin and CD31 microvessel counts $(r=0.54, P=0.002)$. In this group, immunohistochemical markers for angiogenesis, endoglin and CD31, and VEGF did not show significant correlation with other conventional prognostic factors, such as tumor size, grade and depth of invasion.

\section{Patients with Lymph Nodes Metastases}

This group included 28 female and 22 male patients with a mean patient age of $70 \pm 11$ years and a range of 42-88 years. The anatomic location of the resected tumors included 27 cases in right colon and 23 in sigmoid colon. The mean number of lymph nodes retrieved per case was $20 \pm 6$ with a mode of 15 lymph nodes. Histologically, 37 cases were moderately differentiated and 13 were poorly differentiated. Angiolymphatic invasion was identified in 23 cases. Pericolonic lymph nodes involvement was identified in all patients of this group without evidence of distant metastases. Positive vascular pedicle lymph node was identified in two cases. The mean microvessel density value was $31 \pm 5 / 0.15 \mathrm{~mm}^{2}$ field for endoglin, $20 \pm 6 / 0.15 \mathrm{~mm}^{2}$ field for CD31 and 2.2 \pm 1.1 for VEGF. Paired ' $t$ ' test showed a significant difference between mean values of endoglin and CD31 microvessel counts $(P<0.001)$. In this group, only endoglin microvessel counts showed significant correlation with vascular pedicle lymph node status $(r=0.49, P=0.01)$ and grade of the tumor $(r=0.38, P=0.04)$.

\section{Patients with Liver Metastases}

This group included 21 female and 29 male patient with a mean age of $64 \pm 11$ years and a range of 3585 years. The anatomic location of the resected tumors included 33 cases in right colon, four in transverse colon and 13 in sigmoid colon. The mean number of lymph nodes retrieved per case was $22 \pm 10$ with a mode of 13 lymph nodes. Histologically, 35 cases were moderately differentiated and 15 poorly differentiated. Angiolymphatic invasion was identified in 24 cases. Positive pericolonic lymph nodes were identified in 38 cases and vascular pedicle lymph nodes were found in 17 cases. The mean microvessel density value for endoglin was $35 \pm 10 / 0.15 \mathrm{~mm}^{2}$ field, $22 \pm 10 /$ $0.15 \mathrm{~mm}^{2}$ field for CD31 and 2.6 \pm 1.0 for VEGF. Paired ' $t$ ' test showed a significant difference between mean values of endoglin and CD31 microvessel counts $(P<0.001)$. However, there was a significant correlation between endoglin and CD31 microvessel counts $(r=0.48, P=0.001)$. In this group, only endoglin microvessel counts showed significant correlation with vascular pedicle lymph node status $(r=0.43, P=0.03)$ and grade of the tumor $(r=0.45, P=0.018)$.

\section{Total Group (150 patients)}

The mean number of lymph nodes retrieved per case was $22 \pm 9$ with a mode of 15 , for positive lymph node cases, and $19 \pm 7$ and 20 for negative lymph

Table 1 Clinical findings in patients included in the study

Patients

Total (150 cases)

\begin{tabular}{|c|c|c|c|c|}
\hline & Without LN met. & With LN met. & With liver met. & \\
\hline Gender & $15 \mathrm{M} / 35 \mathrm{~F}$ & $22 \mathrm{M} / 28 \mathrm{~F}$ & $29 \mathrm{M} / 21 \mathrm{~F}$ & - \\
\hline \multicolumn{5}{|l|}{ Age } \\
\hline Range (years) & $40-88$ & $42-88$ & $35-85$ & - \\
\hline Mean (years) & $72 \pm 9$ & $70 \pm 11$ & $64 \pm 11$ & - \\
\hline \multirow[t]{3}{*}{ Grade } & $4 \mathrm{WD}$ & $37 \mathrm{MD}$ & $35 \mathrm{MD}$ & - \\
\hline & $41 \mathrm{MD}$ & $13 \mathrm{PD}$ & $15 \mathrm{PD}$ & - \\
\hline & $5 \mathrm{PD}$ & - & - & - \\
\hline Angiolymphatic & $0 / 50$ & $23 / 50$ & $24 / 50$ & - \\
\hline Endoglin ${ }^{\mathrm{a}}$ & $26 \pm 10$ & $31 \pm 5$ & $35 \pm 10$ & $31 \pm 10$ \\
\hline CD31 ${ }^{\mathrm{a}}$ & $15 \pm 8$ & $20 \pm 6$ & $22 \pm 10$ & $19 \pm 8$ \\
\hline VEGF & $1.6 \pm 1.1$ & $2.2 \pm 1.1$ & $2.6 \pm 1.0$ & $2.4 \pm 0.7$ \\
\hline
\end{tabular}

$\mathrm{LN}=$ lymph node; met. = metastases; $\mathrm{F}=$ females; $\mathrm{M}=$ males; $\mathrm{WD}=$ well differentiated; $\mathrm{MD}=$ moderately differentiated; $\mathrm{PD}=$ poorly differentiated.

Count per $\times 400$ magnification $\left(=0.15 \mathrm{~mm}^{2}\right.$ field $)$. 
node cases, without a significant difference. Endoglin stained the neoformed tumor microvessels in every case (150 cases), and CD31 stained only 145 cases (negative in five cases). The mean number of microvessel identified with endoglin was significantly different from the mean number of microvessel identified by CD31 $(31 \pm 10$ and $19 \pm 8 /$ $0.15 \mathrm{~mm}^{2}$ field, respectively, $P<0.001$ ) (Figures 1 and 2). However, there was a significant correlation between microvessel counts by endoglin and CD31 $(r=0.49, P<0.001)$. In addition, CD31 stained the endothelial cells of blood vessels in non-neoplastic tissue, while endoglin was negative (Figures 3 and 4).

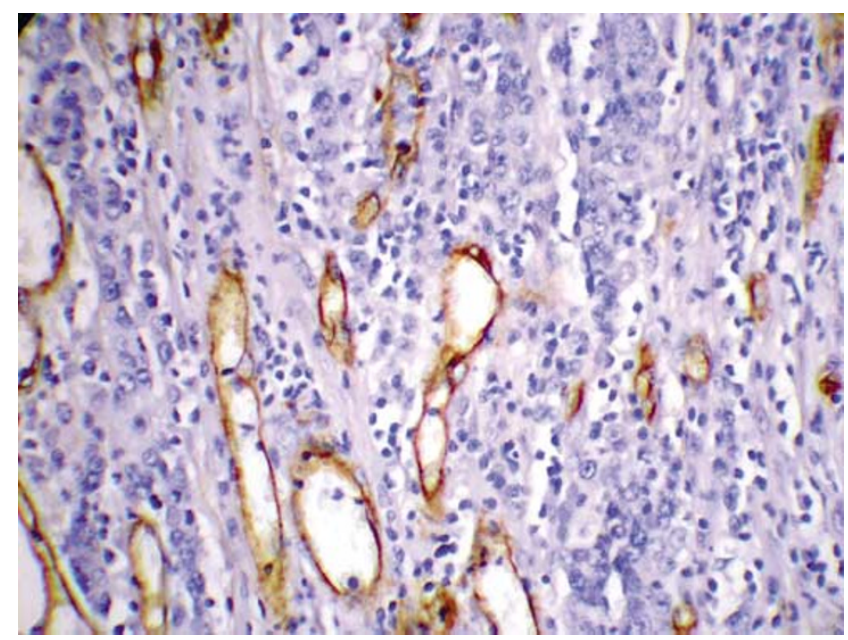

Figure 1 Endoglin expression in a poorly differentiated colonic adenocarcinoma. Note that endoglin staining is stronger in intensity and stains more microvessels than CD31. (Original magnification $\times 400$ ).

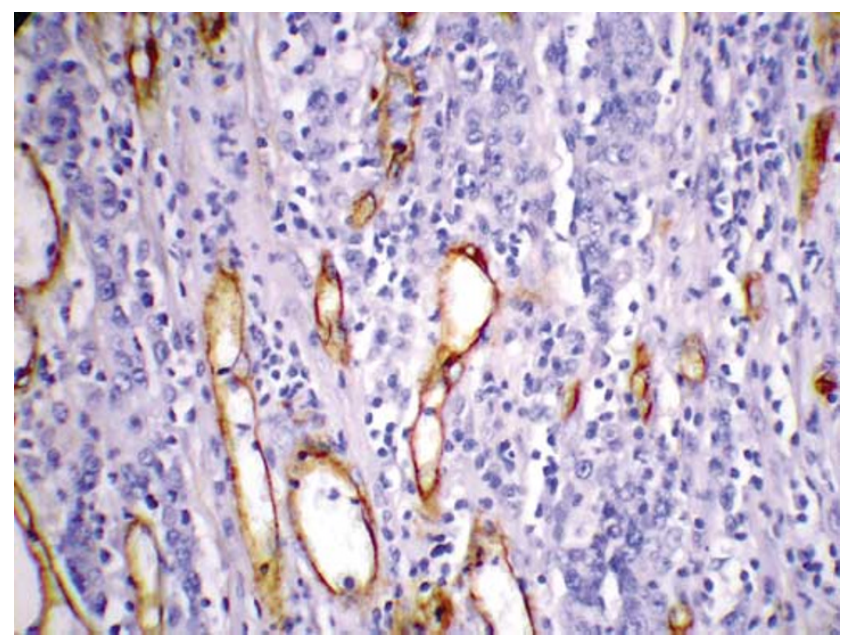

Figure 2 CD31 expression in a poorly differentiated colonic adenocarcinoma as shown in Figure 1. Note CD31 staining is less intense with less number of microvessels. (Original magnification $\times 400$ ).
Although both endoglin and CD31 microvessel counts correlated with the presence of angiolymphatic invasion $(r=0.30, P=0.008$ for endoglin; $r=0.23, P=0.05$ for CD31) and pericolonic lymph node metastases $(r=34, P=0.001$ for endoglin; $r=0.23, P=0.05$ for CD31), only endoglin microvessel counts correlated significantly with liver metastases $(r=0.31, P=0.006)$ and positive vascular pedicle lymph nodes $(r=0.29, P=0.013)$. Using multivariate analysis, endoglin correlated significantly with angiolymphatic invasion and lymph node metastases independent of tumor stage. No significant correlation was identified between endoglin microvessel counts and tumor size $(r=0.18$, $P=0.1$ for endoglin), but CD31 showed a significant correlation ( $r=0.34, P=0.002)$. None of our immunohistochemical markers (endoglin and CD31) showed significant correlation between microvessel counts and grading of the tumor $(r=0.02, P=0.86$ for CD31 and $r=0.11, P=0.33$ for endoglin).

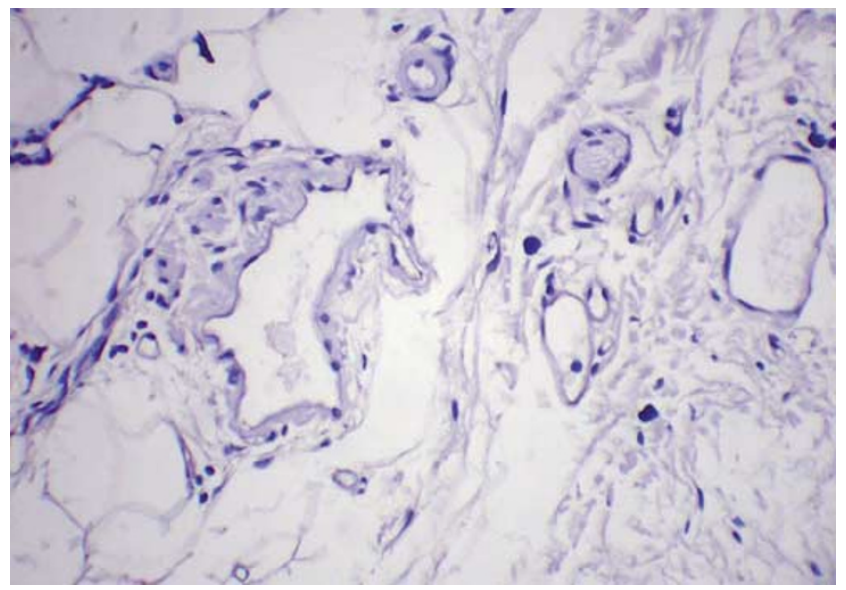

Figure 3 Endoglin does not stain blood vessels in benign colonic tissue (stains only microvessels confined to the tumor). (Original magnification $\times 400$ ).

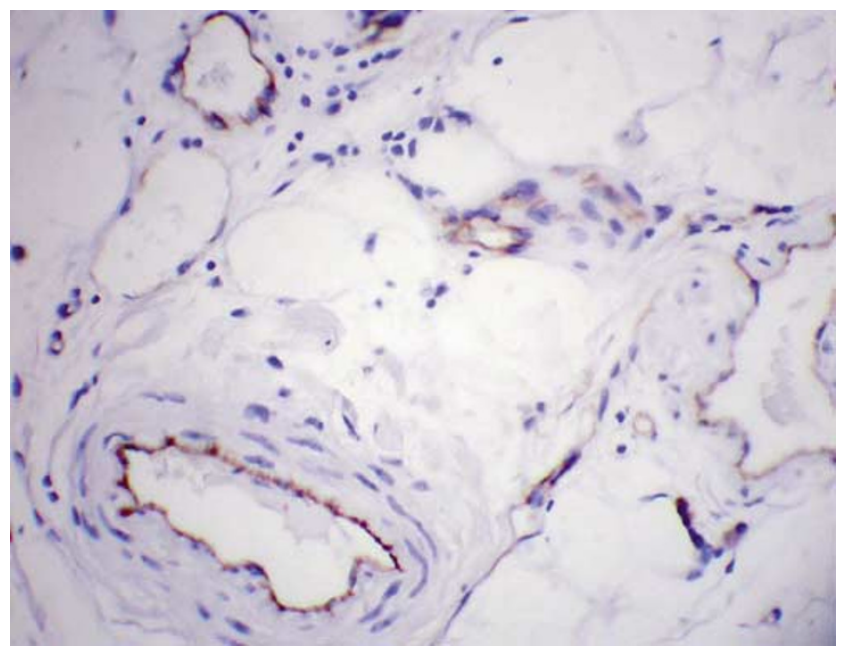

Figure 4 CD31 stains also nonproliferating blood vessels in the colonic tissue. (Original magnification $\times 400$ ). 


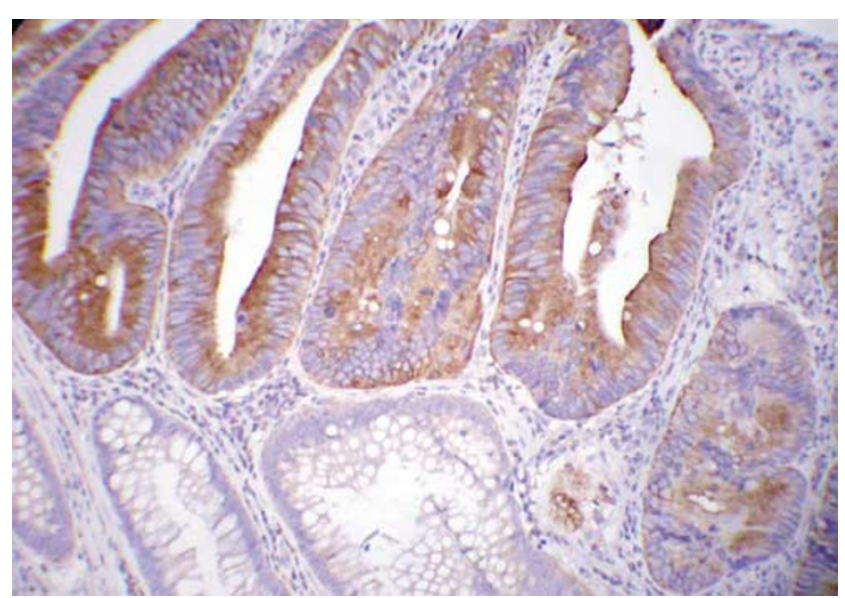

Figure 5 VEGF immunoreactivity in colonic cancer. Note that the benign glands are VEGF negative, while the carcinoma component is strongly positive. (Original magnification $\times 400$ ).

High VEGF expression of the neoplastic cells was identified in 101 cases (67\%) and low or no expression in 49 cases (33\%) (Figure 5). VEGF showed a mean value of $2.4 \pm 0.7$ for colorectal cancer patients and had a significant correlation with angiolymphatic invasion $(r=0.32$, $P=0.002)$, positive pericolonic lymph nodes $(r=0.26, P=0.009)$ and depth of invasion $(r=0.27$, $P=0.007)$. No significant correlation was found between VEGF and grade of tumor $(r=0.13$, $P=0.12)$, vascular pedicle lymph node $(r=0.16$, $P=0.08)$ and liver metastases $(r=0.17, P=0.06)$.

\section{Discussion}

There have been number of studies reporting an association between increased angiogenesis and an increased incidence of metastases with subsequent decreased survival in a variety of malignancies. ${ }^{10-16}$ Previous studies have explored the prognostic value of microvessel counts in colorectal carcinoma, however, with contradictory results. ${ }^{17-20}$

In the current study, one histologic section from each case was immunostained and underwent microvessel counting by two investigators who were blinded to diagnosis and the immunohistochemical markers to better standardize the result. Also, microvessels were only counted in intratumoral 'hotspots' areas. In addition, we only used the high magnification $(\times 400)$ for microvessel identification, allowing more precise quantitation of the number of vessels, which may also explain the higher values obtained in our study compared with the results of previous quantitative studies. ${ }^{19,20,26-28}$

Previous studies used panendothelial markers, namely CD34, CD 31, and von Willebrand factor, to evaluate microvessel density. However, assessing microvessel density with these markers may not be accurate, since they are also expressed in normal vessels. ${ }^{29}$ In addition, these panendothelial markers are not always expressed in all tumor vessels. ${ }^{29,30}$ Factor-VIII stains large vessels with high sensitivity and capillaries with variable and focal staining. ${ }^{31}$ It is also not specific for blood vessels, since it can stain lymphatics, ${ }^{32}$ and some tumor vessels do not stain with Factor-VIII. ${ }^{32}$ Transmembrane glucoprotein CD31 (platelet-endothelial cell adhesion molecule) is found on endothelial cells and many hematopoietic cells. ${ }^{31,33}$ Although it is a good marker for endothelial cells, it stains both large and small vessels with equal intensity, as well as blood vessels in normal and tumor tissue, and occasionally stains carcinoma cells. ${ }^{33}$ CD31 can be negative in intratumoral neovascularization as reported by Giatromanolake et $a l^{34}$ and collaborated in our study (five cases were negative). The reliability of CD31 staining has been inconsistent between laboratories. ${ }^{35}$ The addition of a microwave antigen retrieval step has reduced, but not eliminated, this inconsistency. ${ }^{21} \mathrm{CD} 34$, another transmembrane protein, is found on the surface of endothelial cells, especially those undergoing active angiogenesis, ${ }^{36}$ but specificity is compromised since it can also stain a variety of other mesenchymal cells. ${ }^{37,38}$ Antibody to the $\mathrm{H}$ blood group antigen, such as the monoclonal antibody BNH 9, and Ulex europaeus lectin are also limited by positive staining of carcinoma cells. ${ }^{36}$ Therefore, microvessel quantification with antibodies preferentially expressed only in angiogenic vessels might be more valuable.

In our study, endoglin microvessel staining was consistently present in all the cases studied. Endoglin stained small vessels with high sensitivity in or around the tumor, but the blood vessels in nonneoplastic tissue did not or only weakly stained with endoglin. This is in agreement with previous studies where endoglin was expressed mainly in proliferating blood vessels, while CD34 or CD31 stained all the blood vessels, ${ }^{21,22,24,31}$ suggesting that endoglin is preferentially expressed in vessels undergoing neoangiogenesis. Endoglin is very selective for the blood vessel endothelium and reacts specifically with endothelial cells without significant staining of inflammatory or stromal cells within the neoplasm. ${ }^{22,38}$ Therefore, endoglin staining should reduce false-positive staining of blood vessels when compared with other commonly used panendothelial markers. Another important aspect of this marker is that it can be readily performed on formalin-fixed, paraffin-embedded tissues.

Some authors suggest a specific detection method for endoglin and reported a superior sensitivity using other than standard immunohistochemical method performed on paraffin-embedded tissue sections. ${ }^{22}$ The experience in our laboratory shows that endoglin staining using standard immunohistochemical method still detects more blood vessels than the other panendothelial markers. 
Tumor grade, depth of invasion and presence of angiolymphatic invasion are parameters that correlate with metastases and survival. ${ }^{18}$ Our results demonstrated that using either CD31 or endoglin, microvessel counts in colorectal carcinoma correlated significantly with the presence of angiolymphatic invasion and lymph node metastases, consistent with previous reports. ${ }^{26,39}$ Moreover, microvessel counts by endoglin showed a statistically significant correlation with the presence of vascular pedicle lymph nodes and liver metastases. Using multivariate analysis, endoglin microvessel counts significantly correlated with angiolymphatic invasion, lymph node and liver metastases, independent of tumor stage. This is consistent with recent studies that showed that microvessel counts is an independent prognostic factor. ${ }^{23,24,40}$ CD31 did not show the same correlation. The correlation between microvessel counts and metastatic disease of the colon has been reported. ${ }^{40-42}$ These results are in accordance with angiogenesis being a crucial factor in the metastatic process and thus for the progression of a malignant disease. ${ }^{41}$

Bossi et $a 1^{17}$ and Pietra et $a l^{18}$ found no correlation between microvessel counts and other prognostic parameters including survival in colorectal carcinoma patients without positive lymph nodes. Our results showed similar findings in the negative lymph node patients, suggesting that angiogenesis does not play a role in this stage of colorectal tumors since neither microvessel count nor VEGF overexpression showed significant correlation with any of conventional prognostic parameters. This may explain the good prognosis of these patients.

We examined the correlation of microvessel count in colorectal carcinoma and tumor size. In our study, CD31 microvessel count correlated significantly with tumor size, while endoglin and VEGF did not show any significant correlation.

VEGF may contribute to angiogenesis by stimulating endothelial cell mitogenesis and inducing microvessel permeability. ${ }^{43}$ Several reports have demonstrated that malignant cells in colorectal cancer produce a number of angiogenic growth factors, including VEGF. ${ }^{44-48}$ Some studies have indicated that VEGF expression is an independent prognostic factor in predicting patient prognosis, ${ }^{4-46}$ while others have reported no such association. ${ }^{47}$ Chung et $a l^{48}$ also showed that VEGF expression was significantly associated with prognosis and hematogenous spread in colorectal carcinoma. Our study showed that VEGF is a useful marker for prognosis by significantly correlating with angiolymphatic invasion, lymph node status and depth of invasion, although it was not an independent prognostic factor.

In conclusion, our results showed that assessing microvessel density using endoglin in the colorectal cancer might be a valuable parameter for predicting patients having an increased risk of developing of metastatic disease. We believe that endoglin, by staining only the proliferating microvessels in colorectal carcinoma, is a more specific and sensitive marker for tumor angiogenesis than the other commonly used panendothelial markers. Endoglin staining also showed prognostic significance with positive correlation with the presence of angiolymphatic invasion, lymph node metastases, tumor stage and hepatic metastases, and could possibly lead to future therapeutic trials with antiangiogenic therapy.

\section{Acknowledgements}

This work was presented in part at the 2003 annual meeting of the United States and Canadian Academy of Pathology.

\section{References}

1 Lindmark G, Gerdin B, Pahlman L, et al. Prognostic predictors in colorectal cancer. Dis Colon Rectum 1994;37:1219-1227.

2 Compton C, Fenoglio-Preiser CM, Pettigrew N, et al. American Joint Committee on Cancer Prognostic Factors Consensus Conference: Colorectal Working Group. Cancer 2000;88:1739-1757.

3 Sokmen S, Sarioglu S, Fuzun M, et al. Prognostic significance of angiogenesis in rectal cancer: a morphometric investigation. Anticancer Res 2001;21: 4341-4348.

4 Wolmark N, Fisher B, Wieand HS. The prognostic value of the modifications of the Dukes' C class of colorectal cancer: an analysis of the NSABP trials. Ann Surg 1986;302:115-122.

5 Folkman J. What is the evidence that tumors are angiogenesis dependent? J Natl Cancer Inst 1990;82: 4-6.

6 Folkman J. Angiogenesis and breast cancer. J Clin Oncol 1994;12:441-443.

7 Weidner N. Intratumor microvessel density as a prognostic factor in cancer. Am J Pathol 1995;147:9-19.

8 Polverini PJ, Leibovich SJ. Induction of neovascularization in vivo and endothelial proliferation in vitro by tumor associated macrophages. Lab Invest 1984; 51:635-642.

9 Zatterstrom UK, Brun E, Willen R, et al. Tumor angiogenesis and prognosis in squamous cell carcinoma of the head and neck. Head Neck 1995;17:312-318.

10 Srivastava A, Laidler P, Davies RP, et al. The prognostic significance of tumor vascularity in intermediate thickness (0.76-4.0 mm thick) skin melanoma. A quantitative histologic study. Am J Pathol 1988; 133:419-423.

11 Macchiarini P, Fontani G, Hardin MJ, et al. Relation of neovascularization to metastasis of non-small cell lung cancer. Lancet 1992;340:145-146.

12 Weidner N, Semple JP, Welch WR, et al. Tumor angiogenesis and metastasis: Correlation in invasive breast carcinoma. N Engl J Med 1991;324:1-8.

13 Dickinson AJ, Fox SB, Persad RA, et al. Quantification of angiogenesis as an independent predictor of prognosis in invasive bladder carcinoma. $\mathrm{Br} \mathrm{J}$ Urol 1994;74:762-766. 
14 Weidner N, Carroll PR, Flax J, et al. Tumor angiogenesis correlates with metastasis in invasive prostate carcinoma. Am J Pathol 1993;143:401-409.

15 Wiggins DL, Granai CO, Steinhoff MM, et al. Tumor angiogenesis as a prognostic factor in cervical carcinoma. Gynecol Oncol 1995;56:353-356.

16 Abulafia O, Triest WE, Sherer DM. Angiogenesis in primary and metastatic epithelial ovarian carcinoma. Am J Obstet Gynecol 1997;177:541-547.

17 Bossi P, Viale G, Lee AK, et al. Angiogenesis in colorectal tumors: microvessel quanitation in adenomas and carcinomas with clinicopathological correlations. Cancer Res 1995;55:5049-5053.

18 Pietra N, Sarli L, Caruana P, et al. Is tumor angiogenesis a prognostic factor in patients with colorectal cancer and no involved nodes? Eur J Surg 2000;166:552-556.

19 Saclarides TJ, Speziale NJ, Drab E, et al. Tumor angiogenesis and rectal carcinoma. Dis Colon Rectum 1994;37:921-926.

20 Frank RE, Saclarides TJ, Leurgans S, et al. Tumor angiogenesis as predictor of recurrence and survival in patients with node-negative colon cancer. Ann Surg 1995;222:695-699.

21 Bodey B, Bodey Jr B, Siegel SE, et al. Over-expression of endoglin (CD 105): a marker of breast carcinomainduced neovascularization. Anticancer Res 1998;18: 3621-3628.

22 Brewer CA, Setterdahl JJ, Li MJ, et al. Endoglin expression as a measure of microvessels density in cervical cancer. Obstet Gynecol 2000;96:224-228.

23 Choi HJ, Hyun MS, Jung GJ, et al. Tumor angiogenesis as a predictor in colorectal carcinoma with special reference to mode of metastasis and recurrence. Oncology 1998;55:575-581.

24 Saad RS, Jasnosz KM, Silverman JF. Endoglin (CD105) expression in endometrial carcinoma. Int J Gynecol Pathol 2003;22:248-253.

25 Vermeulen PB, Gasparini G, Fox SB, et al. Quantification of angiogenesis in solid human tumors: an international consensus on the methodology and criteria of evaluation. Eur J Cancer 1996;32:2474-2484.

26 Takebayashi Y, Akiyama S, Yamada K, et al. Angiogenesis as an unfavorable prognostic factor in human colorectal carcinoma. Cancer 1996;78:226-231.

27 Engel CJ, Bennet ST, Chambers AF, et al. Tumor angiogenesis predicts recurrence in invasive colorectal cancer when controlled for Dukes staging. Am J Surg Pathol 1996;20:1260-1265.

28 Tomisaki S, Ohno S, Ichiyoshi Y, et al. Microvessel quantification and its possible relation with liver metastases in colorectal cancer. Cancer 1996;77: 1722-1728.

29 Wang JM, Kumar S, Pye D, et al. Breast carcinoma: comparative study of tumor vasculature using two endothelial cell markers. J Natl Cancer Inst 1994; 86:386-388.

30 Parums DV, Cordell JL, Micklem K, et al. JC70: a new monoclonal antibody that detects vascular endothelium associated antigen on routinely processed tissue sections. J Clin Pathol 1990;43:752-757.

31 Akagi K, Ikeda Y, Sumiyoshi Y, et al. Estimation of angiogenesis with anti-CD105 immunostaining in the process of colorectal cancer development. Surgery 2002;131:S109-S113.
32 Guidi AJ, Fischer L, Harris JR, et al. Microvessel density and distribution in ductal carcinoma in situ of the breast. J Natl Cancer Inst 1994;86:614-619.

33 Mietinnen M, Lindenmayer AE, Chaubal A. Endothelial cell markers CD31, CD34, BNH9 antibody to $\mathrm{H}$ - and Y- antigens: evaluation of their specificity and sensitivity in the diagnosis of vascular tumors and comparison with von Willebrand factor. Mod Pathol 1994; 7:82-90.

34 Giatromanolaki A, Sivridis E, Koukourakis MI, et al. Intratumoral angiogenesis: a new prognostic indicator for stage I endometrial adenocarcinoma? Oncol Res 1999;11:205-212.

35 Smith-McCune KK, Weidner N. Demonstration and characterization of the angiogenic properties of cervical dysplasia. Cancer Res 1994;54:800-804.

36 Kuzu I, Bicknell R, Harris AL, et al. Heterogeneity of vascular endothelial cells with revelance to diagnosis of vascular tumors. J Clin Pathol 1992;45:143-148.

37 Lindenmayer AE, Miettinen M. Immunophenotypic features of uterine stromal cells. CD34 expression in endocervical stroma. Virchows Arch 1995;426: 457-460.

38 Saad RS, Dabbs DJ. Endoglin, CD31 and CD34 expression in the breast cancer. Mod Pathol 2001;14:35A (Abstract).

39 Tanigawa N, Amaya H, Matsumura M, et al. Tumor angiogenesis and mode of metastases in patients with colorectal cancer. Cancer Res 1997;57:1043-1046.

40 Giatromanolaki A, Stathopoulos GP, Tsiobanou E, et al. Combined role of angiogenesis, bcl-2 and p53 expression in the prognosis of colorectal cancer. Cancer 1999;86:1421-1430.

41 Vermeulen PB, van den Eynden GG, Huget P, et al. Prospective study of intratumoral microvessel density, p53 expression and survival in colorectal cancer. Br J Cancer 1999;79:316-322.

42 Burrows FJ, Derbyshire EJ, Tazzari PL, et al. Upregulation of endoglin on vascular endothelial cells in human solid tumors: implications for diagnosis and therapy. Clin Cancer Res 1995;1:1623-1634.

43 Claffey KP, Robinson GF. Regulation of VEGF/VPF expression in tumor cells: consequences for tumor growth and metastasis. Cancer Metast Rev 1996;15:165-176.

44 Kang SM, Maeda K, Onoda N. Combined analysis of p53 and vascular endothelial growth factor expression in colorectal carcinoma for determination of tumor vascularity and liver metastasis. Int $\mathrm{J}$ Cancer 1997;74:502-507.

45 Lee JC, Chow NH, Wang ST, et al. Prognostic value of vascular endothelial growth factor expression in colorectal cancer patients. Eur J Cancer 2000;36:748-753.

46 Ishigami SI, Arii S, Furutani M, et al. Predictive value of vascular endothelial growth factor (VEGF) for metastasis and prognosis of human colorectal cancer. Br J Cancer 1998;78:1379-1384.

47 Takahashi Y, Tucker SL, Kitadai Y. Vessel counts and expression of vascular endothelial growth factors as prognostic factors in node-negative colon cancer. Arch Surg 1997;132:541-546.

48 Chung YS, Maeda K, Swa M. Prognostic value of angiogenesis in gastrointestinal tumors. Eur J Cancer 1996;32A:2501-2505 